\section{Focusing on glaucoma progression and the clinical importance of progression rate measurement: a review}

L Rossetti, F Goni, P Denis, B Bengtsson, A Martinez and A Heijl newly developed analyses will be helpful to forecast the potential future development of the glaucoma. An individualized treatment approach then requires that in patients in whom the risk of becoming visually impaired or blind during their lifetime is higher, a more intensive medical IOP-lowering therapy such as fixed combinations can be considered as treatment option.

Eye (2010) 24, S1-S7; doi:10.1038/eye.2010.112

Keywords: glaucoma; progression; rate of progression; visual fields; perimetry; target pressure

\section{Introduction}

The number of people with visual impairment in 2002 was more than 161 million worldwide, of whom about 37 million were bilaterally blind. After cataract glaucoma is found to be the second leading cause of blindness. ${ }^{1}$ The impact of glaucoma on health-care systems is currently increasing with ageing population. In the Western world chronic open-angle glaucoma (OAG) is by far the most common form of the disease.

Disease progression in glaucoma is not uncommon and despite treatment, most patients still progress. A retrospective community-based longitudinal study from Olmsted County in the United States reviewed charts of 295 OAG patients for a mean followup time of 15 years to determine the probability of a patient developing 'legal blindness' in either one or both eyes from newly diagnosed OAG after starting medical or surgical therapy
Department of Ophthalmology, University Hospital, Lund University, Malmö, Sweden

Correspondence: A Heijl, Department of Ophthalmology, University Hospital, Lund University, SE-20502 Malmö, Sweden. Tel: + 4640 332742; Fax: + 4640924221 E-mail: Anders.Heij@@ med.lu.se 
or both. At 20 years follow-up the Kaplan-Meier cumulative probability of glaucoma-related blindness in at least one eye was estimated to be $27 \%$, and $9 \%$ in both eyes. These findings support the assumption that the risk of going blind. ${ }^{2}$

Further analysis from the same study showed that about $20 \%$ of patients progressed to blindness in at least one eye over the 20-year period of the study. Most of these patients had visual field (VF) loss at diagnosis but, more interestingly and importantly, different susceptibilities to intraocular pressure (IOP) were found. Some patients became blind at IOP levels that others could tolerate without significant disease progression. ${ }^{3}$ These findings strongly suggest that continued monitoring of VF and reassessment of target IOPs upon confirmation of VF damage progression are mandatory in the management of glaucoma almost if IOP levels are within the statistical normal limits.

\section{Materials and methods}

The importance of progression detection and rate forms the basis of this review. Its scientific content derives from established and recently published literature sources as well as a series of 2008 congress lectures given at the World Ophthalmology Congress in Hong Kong, China (27 June 2008), the Malmö Progression Meeting in Malmö, Sweden (16-17 March 2008), and the annual meeting of the European Glaucoma Society (EGS) in Berlin, Germany (1-6 June 2008).

\section{Results}

\section{Natural history of glaucoma progression}

Data on the natural history of visual function deficits in glaucoma are scarce, as therapy is normally initiated VF defects diagnosed. Only in specific cases will the treating physician observe without treatment, for example when IOP levels are still within the normal range.

Consequently, prospective natural history data have been reported on patients with normal-tension glaucoma (NTG): the CNTGS study reported a mean progression of $-0.39 \mathrm{~dB}$ per year and $33 \%$ of all patients actually progressed. ${ }^{4}$ Although NTG is common in population studies, these patients are often diagnosed late and are a minority in clinical glaucoma care, except in Japan.

A recent publication presents natural history data not only in NTG, but also in high-tension glaucoma (HTG) as well as pseudoexfoliation glaucoma (PEXG).

This article reports on 118 control patients from the Early Manifest Glaucoma Trial (EMGT), who were followed for at least 6 years without treatment or progressed within 6 years. Data were only included until the patient progressed, because therapy could be introduced at that point in time. Rate of progression essentially followed the same pattern, but differed significantly among the three subgroups. Although the mean overall progression rate was $-1.08 \mathrm{~dB}$ per year (SD \pm 2.07 ), the values for HTG, NTG, and PEXG were -1.31 (SD \pm 1.93$),-0.36(\mathrm{SD} \pm 0.94)$ and $-3.13 \mathrm{~dB}$ $(\mathrm{SD} \pm 3.69)$ respectively. The progression among PEXG patients was very fast. Progression appeared to be considerably and significantly faster in older patients (median rate, $-1.48 \mathrm{~dB}$ per year) than in younger ones (median rate, $-0.60 \mathrm{~dB}$ per year). Based on these data, the mean rate to progress from normal visual function to blindness is approximately 25 years, with PEXG patients progressing considerably faster than NTG HTG patients. The authors therefore advise to look for signs of PEXG in screening and glaucoma case finding.

Such knowledge of the natural, untreated rate of glaucoma progression could allow estimates of the amount of damage arising if access to glaucoma screening is delayed or not offered at certain intervals, or if start of clinical care is delayed. ${ }^{5}$

\section{Risk factors for disease progression in glaucoma}

The growing evidence about the wide variability in disease progression and outcomes between patients means that it is essential to study the risk factors that influence this variability. More knowledge in this field will help the clinician to identify the patients who require more care and might need a more aggressive treatment to achieve a better outcome.

The clinical importance of measuring VF progression rates and the clinical use of progression rate for making management decisions are now well recognized. Reviewing data from key glaucoma studies available to date such as EMGT, CIGTS, AGIS, OHTS, and EGPS (Table 1) leads to the following main conclusions: although major glaucoma studies confirmed elevated IOP as a major risk factor, they differed in identifying additional risk factors such as VF damage, age, pseudoexfoliation, or presence of disc haemorrhages.

All trials, but CNTGS, gave evidence for the importance of IOP reduction in management of OHT/POAG.

Within the Canadian Glaucoma Study, a multi-centre prospective longitudinal study of 258 patients with OAG, independent factors, predictive for glaucomatous field progression, were identified: higher baseline age (hazard ratio per each additional year of age: 1.04), female gender (hazard ratio 1.94), higher mean follow-up IOP (hazard ratio per $1 \mathrm{~mm} \mathrm{Hg} \mathrm{1.19),} \mathrm{and} \mathrm{abnormal}$ baseline anti-cardiolipin antibody levels (hazard ratio 
Table 1 Summary of risk factors identified in major glaucoma progression studies

\begin{tabular}{lccccc}
\hline Study & EMGT & CNTGS & AGIS & OHTS & EGPS \\
\hline IOP & $\sqrt{ }$ & - & $\sqrt{ }$ & $\sqrt{ }$ & $\sqrt{ }$ \\
Damage & $\sqrt{ }$ & - & $\sqrt{ }$ & NA & NA \\
Age & $\sqrt{ }$ & - & $\sqrt{ }$ & $\sqrt{ }$ & $\sqrt{ }$ \\
Exfoliations & $\sqrt{ }$ & $\mathrm{x}$ & $\mathrm{x}$ & $\mathrm{x}$ & $\sqrt{ }$ \\
Disc haemorrhages & $\sqrt{ }$ & $\sqrt{ }$ & $\mathrm{x}$ & $\sqrt{ }$ & $\mathrm{x}$ \\
\hline
\end{tabular}

Abbreviations: $\sqrt{ }$, identified as risk factor; - , not identified as risk factor; $x$, data not collected/analyzed; NA, parameter not applicable.

3.86). IOP was found to be a powerful predictor of progression, with 19\% increase of risk for every $1 \mathrm{~mm} \mathrm{Hg}$ increase in mean IOP. ${ }^{6}$

Glaucoma progression rates are highly variable among patients and unfortunately risk factors explain only part of this variation. Therefore, target IOP needs to be individualized and in case of a significant progression a lower target should be sought. After a sufficient number of VFs and when rate of progression is known, it should be the main factor to set or adjust the target IOP. This approach should result in an earlier and a more aggressive treatment of at risk patients, but will also mean that patients with no direct risk for a faster progressive disease can be managed with less frequent visits and with higher target IOP. In case of a further significant progression, treatment should be adjusted and a lower target IOP be set. As the aim of any further intervention is to change the course of the disease reducing the rate of progression, the frequency of VF testing should be consequently increased to detect slower rates of progression with sufficient power, as discussed below.

Figure 1 shows examples of a faster and a more slowly progressing patient. If values are plotted against the patient's age, the clinician may assess if this particular patient is potentially at risk of becoming blind.

These concepts/ideas are also highlighted in the 2008 EGS guidelines. The recent guidelines recommend the full integration of VF progression rates into clinical glaucoma practice. ${ }^{7}$

\section{Impact of glaucoma progression on patient quality of life}

Glaucoma is a chronic disease that is not life threatening but can have a substantial negative impact on patients' quality of life (QoL) when leading to partial or total loss of sight. In recent years a number of studies have been trying to document the extent of sight-related impact on a patient's life. The following section highlights major findings from recent years.
Visual field loss and disability

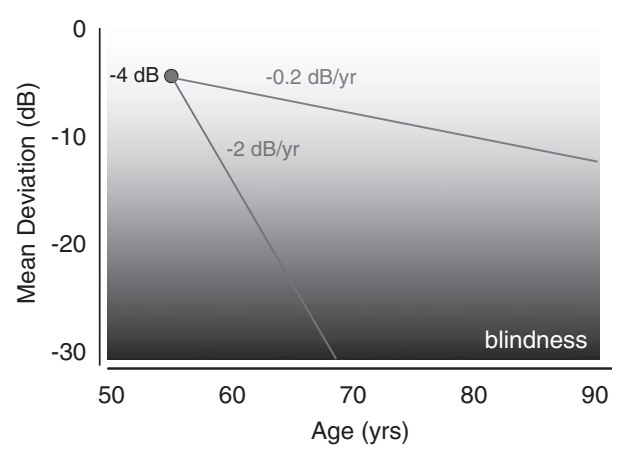

Figure 1 Example of different VF deterioration rates in a glaucoma patient. Depending on rate of progression/steepness of slope a patient may become blind or visually impaired during his or her lifetime.

General aspects in vision-related QoL

White $e t a l^{8}$ found glaucoma to be a major risk factor for occurrence of hip fractures both in men and women with a hazard ratio of 1.9 and 1.3 respectively. The consequences of such fractures in the elderly may present a major impact on mobility and thus QoL.

Functional consequences in eye-hand coordination arising from glaucoma have also been reported in glaucoma patients. Patients were compared with an age-matched control group without any ocular diseases. Glaucoma patients showed statistically significant delays in average movement onset $(P<0.0001)$ and overall movement time $(P<0.5)$, suggesting impairments in initial movement planning and control. Deficits were also recorded in the reaching component, suggesting that glaucoma patients made more tentative movements when reaching out for the object. These deficits were correlated with increasing severity in VF defect as well as impaired stereoacuity. ${ }^{9}$

Kobelt $e t a l^{10}$ analysed health outcomes and QoL of 199 Swedish patients with ocular hypertension or OAG. Patients were grouped into five severity stages based on the VF defect values. Findings in this pilot study showed that the VF in the better-seeing eye was significantly correlated with patients' utility in advanced glaucoma. A significant correlation was also found between total visual acuity (VA) and QoL, and VF (measured mean deviation, MD) in the worse eye was correlated with total VA. ${ }^{10}$ This means that glaucoma patients with advanced vision loss experience a marked reduction in their QoL.

A different study reported the costs and QoL of late stage primary OAG in four European markets. Even though the study was limited by its observational, uncontrolled design, the authors found that QoL was positively correlated with the level of vision in the betterseeing eye, that is QoL worsened as vision decreased. ${ }^{11}$ A study by Aspinall et $a l^{12}$ analysed the QoL in glaucoma 
patients and found that patients rated outdoor mobility and tasks associated with central vision as most important for their personal freedom and QoL. Outdoor mobility was shown to decrease significantly with increasing field loss. ${ }^{12}$ A systematic literature review compared the QoL impact of glaucoma to other chronic diseases such as osteoporosis, type 2 -diabetes, and dementia. The authors found that glaucoma affects patients' QoL as much as other chronic but currently more prevalent diseases. ${ }^{13}$ In 2009, Evans et al ${ }^{14}$ summarized the findings of a systematic literature review on the QoL impact of peripheral vision loss (PVL, with a focus on glaucoma) vs central vision loss (CVL, with a focus on age-related macular degeneration). The results showed that overall more mental than physical components were affected in glaucoma. Vitality and general health were affected substantially. Glaucoma also seemed to affect patients' expectations of treatment and their general vision. Overall the impact on QoL was found to be comparable between PVL and CVL. ${ }^{14}$

Quantifiable milestones in vision-related QoL: eligibility to drive a car and disability pension

Because individual patients set different priorities and define different activities as important in their daily lives, most QoL parameters are essentially 'soft' indicators for the disease impact on QoL. There are however more precise definitions that may be used as milestones to characterize the impact of glaucoma.

Once permission to drive is withdrawn patients normally also lose part of their mobility and independence, making it difficult to obtain essential services and daily goods. ${ }^{15}$ They also reduce their frequency of contact with relatives and friends as social opportunities become more restricted. ${ }^{16}$

Rules and regulations defining fitness to drive differ between countries.

Visual acuity limits are present in all drivers' license regulations in most countries it is 0.5 . VF limits are included in some drivers' license regulations.

Binocular VF testing is not routinely used in clinical testing and management of glaucoma, but it is likely of more value than monocular testing in the assessment of functional ability in activities of daily living and to describe visual disability. ${ }^{17}$

Although the patient's inability to drive a car is one quantifiable milestone in the daily life of a glaucoma patient, another one is the eligibility to disability pension based the status of 'low vision'. Requirements to obtain disability pensions due to low or absent vision were found to be unique for each country. There are multiple layers of rules and requirements that apply to define 'legal blindness'. Some countries as well as the official WHO criteria distinguish between partially sighted and blind, whereas in others no such separation exists. As a result disability pension requirements cannot be standardized in way that would allow comparability between countries. ${ }^{18}$

\section{Quantification of VF progression rate}

Practical guidance for measuring VF progression rate The objective of guidelines (such as the EGS guidelines 2008) is to educate ophthalmologists on management of glaucoma. The most recently published guidelines include a recommendation for use of perimetry to measure rate of disease progression. The guidelines do no longer emphasize detection but the importance of measuring rate of VF progression also in daily clinical practice. ${ }^{19}$

The guidelines issue recommendations similar to those reported by Chauhan and colleagues, which discuss with a given number of exams, how factors such as age at baseline examination and rate of progression should be used for analysis. Recommendations the number and frequency of VF measurements, are discussed. A patient $-2 \mathrm{~dB}$ per year (a high progression rate) has a high risk have visual disability in his lifetime even if has glaucoma with VF of $-4 \mathrm{~dB}$. The data suggest that if newly diagnosed patients have 5-6 VF measures in the first 2-3 years, the treating physician is almost certain to detect if the patient is progressing rapidly (in this case $-2 \mathrm{~dB}$ per year) and at potential risk of visual disability.

Recommended to repeat testing every 4 months during the first 2 years after diagnosis or to test twice per year for the first 3 years. An estimate of progression can then be extrapolated to the patient's life expectancy and management can be changed if the patient is at risk of disability.

Other tests should not be performed at the expense of standard automated perimetry (SAP).

Use of short-wavelength automated perimetry and frequency doubling technology, retinal nerve fibre layer, or optic disc examinations is not as suited to measure rates of VF progression. ${ }^{20}$

\section{New software for predicting rate of VF progression over time}

Bengtsson and Heij ${ }^{21}$ developed a new visual field index (VFI). It was developed for quantifying perimetric rate of progression with SAP, and has been implemented in the Humphrey Field Analyzer (Carl Zeiss Meditec Inc., Dublin, CA, USA). ${ }^{21}$ VFI replaces the previous unit MD measured in $\mathrm{dB}$ with a more comprehensible unit defined as percentage of a full field where $100 \%$ represents a normal VF and $0 \%$ represents a perimetrically blind field. VFI is plotted against the age of the patient. The rate of progression is then calculated 
by linear regression analysis and presented as yearly change in the VFI. The current trend is extrapolated if enough reliable data are available (five tests or more) over a time period of 2 years or more. Rate of progression with VFI is considerably more resistant to the disturbing effect of increasing cataract than the currently used MD value. $^{22}$

This software is a very useful clinical tool for helping the clinician to manage the patients more efficiently printouts are more compact, that is all relevant data are summarized on one page; two baseline tests, the most recent test together with its glaucoma change probability map indicating test point locations with significant deterioration and indicating whether the field shows no, possible, or likely progression compared with the two baseline tests, and the rate of progression analysis.

A pilot evaluation comparing the performance of VFI with MD in calculating the rate of glaucomatous progression was conducted in three groups of glaucoma patients: eyes with developing cataract, eyes with no cataract, and eyes where cataract surgery was performed in the middle of the series. It was concluded that the progression rates calculated using VFI seem to be considerably less affected by cataract and cataract surgery compared with the rates based on the traditional MD. ${ }^{5}$

Short-term progression rates can also be used to predict long-term VF outcomes. VF progression rates were calculated from 100 patients with at least $10 \mathrm{VF}$ tests over an average period of about 8 years. Final VFI was predicted based on the first five VF test results and also by using all viable test results from the observation period. Median VFI progression rate was found to be $-1.1 \%$ per year using both approaches. The authors concluded that information from the first five VF tests was a reliable predictor future VF loss in most patients. ${ }^{23}$

\section{Treatment decisions based on the patient's individual rate of progression}

Findings from the EMGT provide insight into the relationship between glaucoma progression and the effect of treatment. Mainly through population screening, the investigators identified and included 255 patients with early glaucoma, and a median IOP of $20 \mathrm{~mm} \mathrm{Hg}$. Patients were randomized to either laser trabeculoplasty and topical betaxolol or no initial treatment. The magnitude of initial IOP reduction was a major factor influencing outcome. On average, treatment reduced IOP by $25 \%$ (or $5.1 \mathrm{~mm} \mathrm{Hg}$ ), a reduction maintained throughout follow-up. Each higher millimetre of mercury IOP on follow-up was associated with an approximate $13 \%$ increased risk of progression. Progression varied between patient categories and was more common in the control group at any point during the follow-up periods. ${ }^{24,25}$

Guidelines for medical treatment as defined by the EGS with special focus on fixed timolol-prostaglandin combinations European Glaucoma Society guidelines define the goal of the ophthalmologists as 'to maintain the patient's quality of life and visual function at a sustainable cost'. Focus should be also on monitoring the rate of progression to be able to aim for a good, individual target pressure. Lowering IOP is currently the only proven means of preserving the VF.

One approach aimed at better controlling patients at risk for progression by reaching a lower target IOP is to administer a combination of IOP-lowering medications. If target IOP cannot be achieved by administering monotherapy, a combination of two drugs is recommended. The need to use more than one hypotensive drug complicates the management of glaucoma, but such complications can be reduced by the use of fixed combinations. ${ }^{5}$ In Europe, about one-third of patients are receiving combination therapy. Of these, two-thirds are treated with a fixed combination. Fixed combination therapies allow the simplification of a multi-drug regimen by reducing the total number of drops instilled per day, thus reducing exposure to preservatives and related tolerability issues. Furthermore, fixed combinations of timolol and prostaglandin analogues are usually better tolerated than the concomitant administration of the two components. Timolol may exert its effect at the cellular level to reduce the occurrence of conjunctival hyperaemia. ${ }^{26,27}$ Fixed combinations also avoid the washout effect from rapid sequential instillation of individual drops, and may improve compliance to chronic therapy and a single bottle reduces the risk of confusion between two treatments. The fixed combination of a prostaglandin analogue and a $\beta$-blocker is currently considered to be the most effective eye drop to control IOP. ${ }^{28-30}$

According to the EGS guidelines, fixed combinations have many advantages and may be preferable than two separate instillations of the same agents. ${ }^{5}$

Comparing the effect of different fixed prostaglandin-timolol combinations on intraocular pressure

Several recent studies have determined efficacy of different prostaglandin-timolol fixed combinations. The following study summaries present only a selection of available data:

A 12-week, multi-centre, randomized, double-masked study in $200 \mathrm{POAG} / \mathrm{OHT}$ patients determined the efficacy of bimatoprost, comparing it to a fixed combination of latanoprost and timolol (LTFC) by a 
24-h mean IOP curve. Patients were switched from a non-fixed combination of latanoprost and timolol after not reaching the target IOP. Mean change from baseline IOP was $-0.2 \mathrm{~mm} \mathrm{Hg}$ in the bimatoprost group and $+0.8 \mathrm{~mm} \mathrm{Hg}$ in the LTFC group.

The data presented suggest that bimatoprost is not inferior to the LTFC in maintaining IOP at a controlled level during a 24-h period in patients switched from the non-fixed combination of latanoprost and timolol. Both treatments had a similar side effect profile. ${ }^{31}$

Data from another prospective, randomized, evaluator-masked, single-centre, cross-over, comparative trial on fixed combinations measuring the 12-h diurnal IOP curves of bimatoprost-timolol fixed combination (BTFC) and LTFC were reported in a recent report. A total of 54 patients were included in the study and drug was administered in the evening. The $12 \mathrm{~h}$ mean IOP values were $22 \mathrm{~mm} \mathrm{Hg}$ (SD 1.0) at baseline, $17.7 \mathrm{~mm} \mathrm{Hg}$ (SD 0.8) for BTFC group and $18.5 \mathrm{~mm} \mathrm{Hg}$ (SD 0.8) for the LTFC group. The difference was statistically significant $(P<0.001)$. The data suggest that the evening-dosed BTFC provides better IOP control over $12 \mathrm{~h}$ than LTFC. ${ }^{32}$

A third prospective, multi-centre, randomized, investigator-masked clinical trial was comparing efficacy and tolerability of a once daily evening dose of BTFC and LTFC. A total of 82 patients, not satisfactorily controlled on prostaglandin monotherapy were enrolled into this 12-week study. Although mean baseline IOP was comparable for both groups $(22 \mathrm{~mm} \mathrm{Hg})$, mean IOP reduction was significantly greater in the BTFC compared with the LTFC group (-21.4 vs 13.7\%, $P<0.001)$. The authors concluded that although both combinations were more effective in lowering IOP than monotherapy, BTFC showed a higher performance than LTFC in terms of relative IOP reduction. ${ }^{33}$

Such results emphasize the efficacy and support the place of these drugs in the armamentarium of managing the progressing patient.

\section{Conclusions}

Estimating a patient's individual rate of VF progression by either plotting VF measures into the age-disability (mean deviation) graph or using newly developed software is necessary to determine the potential future development of the glaucoma.

Once the relative risk of the patient to become visually impaired is determined, a decision to an individualized treatment approach should be taken. In patients where the risk of becoming visually impaired or blind during the lifetime is higher, an earlier, more aggressive IOPlowering therapy should be considered, and clinical follow-up to determine the development of glaucoma damage should be particularly vigilant. Within the range of IOP-lowering compounds fixed combinations with their more potent IOP-lowering efficacy and good tolerability and low side effect profile are very suitable for management of the at-risk or the fast progressing patients.

\section{Conflict of interest}

L Rossetti has received consulting fees and lecture fees from Alcon, Allergan, MSD, Oftagen, Pfizer, Sooft, and Visufarma. F Goni has received consulting fees from Alcon, Allergan and Pfizer, and lecture fees from Alcon, Allergan, Pfizer, and Zeiss Meditec. P Denis has received consulting fees and lecture fees from Alcon, Allergan, MSD, and Pfizer. B Bengtsson has received consulting fees from Carl Zeiss Meditec and lecture fees from Allergan. A Martinez has received consulting fees and lecture fees from Allergan and MSD. A Heijl has received consulting fees from Allergan, Carl Zeiss Meditec and Alcon, lecture fees from MSD and Santen, and grant support from Carl Zeiss Meditec.

\section{Acknowledgements}

Medical writing assistance was provided by Patricia Buchholz, Karlsruhe, Germany. All authors have acted as advisors to Allergan Inc. A publication grant was provided by Allergan. This supplement issue was generously sponsored by Allergan Ophthalmology.

\section{References}

1 Resnikoff S, Pascolini D, Etya'ale D, Kocur I, Pararajasegaram R, Pokharel GP et al. Global data on visual impairment in the year 2002. Bull World Health Organ 2004; 82(11): 844-851.

2 Hattenhauer MG, Johnson DH, Ing HH, Herman DC, Hodge DO, Yawn BP et al. The probability of blindness from open-angle glaucoma. Ophthalmology 1998; 105: 2099-2104.

3 Oliver JE, Hattenhauer MG, Herman D, Hodge DO, Kennedy R, Fang-Yen $\mathrm{M}$ et al. Blindness and glaucoma: a comparison of patients progressing to blindness from glaucoma with patients maintaining vision. Am J Ophthalmol 2002; 133(6): 764-772.

4 Collaborative Normal-Tension Glaucoma study Group. Natural history of normal-tension glaucoma. Ophthalmology 2001; 108: 247-253.

5 Heijl A, Bengtsson B, Hyman L, Leske MC, Early Manifest Glaucoma Trial Group. Natural history of open-angle glaucoma. Ophthalmology 2009; 116: 2271-2276.

6 Chauhan B, Mikelberg F, Balaszi AG, LeBlanc RP, Lesk MR, Trope GE, Canadian Glaucoma Study Group. Canadian glaucoma study 2 risk factors for the progression of openangle glaucoma. Arch Ophthalmol 2008; 126(89): 1030-1036.

7 European Glaucoma Society. Terminology and Guidelines for Glaucoma, 3rd edn. Savona: Italy, 2008, pp 21, 82-88. 
8 White SC, Atchison KA, Gornbein JA, Nattiv A, PaganiniHill A, Service SK. Risk factors for fractures in older men and women: the leisure world cohort study. Gender Med 2006; 3(2): 110.

9 Kotecha A, O'Leary N, Melmoth D, Grant S, Crabb DP. Functional consequences of glaucoma for eye-hand coordination. Invest Ophthalmol Vis Sci 2009; 50(1): 203-213.

10 Kobelt G, Jonsson B, Bergström A, Chen E, Lindén C, Alm A. Cost-effectiveness analysis in glaucoma: what drives utility? Results from a pilot study in Sweden. Acta Ophthalmologica Scandinavica 2006; 84: 363-371.

11 Thygesen J, Aagren M, Arnavielle S, Bron A, Fröhlich SJ, Baggesen $\mathrm{K}$ et al. Late stage, primary open-angle glaucoma in Europe: social and health care maintenance costs and quality of life of patients from 4 countries. Curr Med Res Opin 2008; 24(6): 1763-1770.

12 Aspinall PA, Hill AR, Nelson P, O'Brien C, O'Connell E, McCloughan L et al. Quality of life in patients with glaucoma: a conjoint analysis approach. Vis Impair Res 2005; 7: 13-26.

13 Mills T, Law SK, Walt JW, Buchholz PM, Hansen J. The impact of glaucoma on quality of life: comparison with the chronic diseases osteoporosis, type 2 diabetes mellitus, and dementia. Drugs Aging 2009; 26(11): 933-950.

14 Evans K, Law S, Walt J, Buchholz P, Hansen J. The quality of life impact of peripheral versus central vision loss with a focus on glaucoma versus age-related macular degeneration. Clin Ophthalmol 2009; 3: 433-445.

15 Fonda SJ, Wallace RB, Herzog AR. Changes in driving patterns and worsening depressive symptoms among older adults. B Psychol Sci Soc Sci 2001; 56: S343-S351.

16 Mezuk B, Rebok GW. Social integration and social support among older adults following driving cessation. J Gerontol B Psychol Sci Soc Sci 2008; 63: S298-S303.

17 Driver and Vehicle Licensing Agency. For medical practitioners: at a glance. Guide to the current medical standards of fitness to drive. Issued by Drivers Medical Group, DVLA, Swansea, 2008.

18 Bron A, Viswanathan A, Thelen U, Di Natale R, Gundgaard $\mathrm{J}$, Schwartz $\mathrm{G}$ et al. International vision requirements for driver licensing and disability pensions: using a milestone approach in the characterization of progressive eye disease. 2010 (in press).

19 European Glaucoma Society. Terminology and Guidelines for Glaucoma, 3rd ed. Savona, Italy, 2008, pp 138-141.

20 Chauhan BC, Garway-Heath DF, Goni FJ, Rossetti L, Bengtsson B, Viswanathan AC et al. Practical recommendations for measuring rates of visual field change in glaucoma. Br J Ophthalmol 2008; 92: 569-573.
21 Bengtsson B, Heijl A. A visual field index for calculation of glaucoma rate progression. Am J Ophthalmol 2008; 145(2): 191-192.

22 Bengtsson B, Patella M, Heijl A. Prediction of glaucomatous visual field loss by extrapolation of linear trends. Arch Ophthalmol 2009; 127: 1610-1615.

23 Bengtsson B, Patella M, Heijl A. Prediction of glaucomatous visual field loss by extrapolation of linear trends. Arch Ophthalmol 2009; 127: 1610-1615.

24 Heijl A, Leske MC, Bengtsson B, Hyman L, Bengtsson B, Hussein M, Early Manifest Glaucoma Trial Group. Reduction of intraocular pressure and glaucoma progression: results from the Early Manifest Glaucoma Trial. Arch Ophthalmol 2002; 120(10): 1268-1279.

25 Leske MC, Heijl A, Hussein M. Factors for glaucoma progression and the effect of treatment. The Early Manifest Glaucoma Trial. Arch Ophthamol 2003; 121: 52.

26 Barnebey H, Orengo-Nania S, Flowers BE, Samples J, Mallick S, Landry TA et al. The safety and efficacy of travoprost $0.004 \% /$ timolol $0.5 \%$ fixed combination ophthalmic solution. Am J Ophthalmol 2005; 140: 1-7.

27 Hommer A, Ganfort Investigators Group I. A doublemasked, randomized, parallel comparison of a fixed combination of bimatoprost $0.03 \%$ /timolol $0.5 \%$ with nonfixed combination use in patients with glaucoma or ocular hypertension. Eur J Ophthalmol 2007; 17: 53-62.

28 Jaenen N, Baudouin C, Pouliquen P, Manni G, Figueiredo A, Zeyen T. Ocular symptoms and signs with preserved and preservative-free glaucoma medications. Eur J Ophthalmol 2007; 17: 341-349.

29 Gurwitz JH, Glynn RJ, Monane M, Everitt DE, Gilden D, Smith $\mathrm{N}$ et al. Treatment for glaucoma: adherence by the elderly. Am J Pub Health 1993; 83(5): 711.

30 Patel S, Spaeth G. Compliance in patients prescribed eye drops in glaucoma. Ophthalmic Surg 1995; 26(3): 234-235.

31 Rossetti L, Karabatsas C, Topouzis F, Vetrugno M, Centofanti M, Boehm A. Comparison of the effect of bimatoprost and a fixed combination of latanoprost and timolol on circadian intraocular pressure. Ophthalmology 2007; 114: 2244-2251.

32 Martinez A, Sanchez M. Bimatoprost/timolol fixed combination versus latanoprost/timolol fixed combination in open angle glaucoma patients. Eye 2009; 23: 810-818.

33 Centofanti M, Oddone F, Vetrugno M, Manni G, Fogagnolo P, Tanga L et al. Efficacy of fixed combinations of bimatoprost or latanoprost plus timolol in patients uncontrolled with prostaglandin monotherapy: a multicentre, randomized, investigator-masked, clinical study. Eur J Ophthalmol 2009; 19(1): 66-71. 